\title{
The AMS-02 detector on the International Space Station - The status after the first 5 years on orbit
}

\author{
Matteo Duranti ${ }^{1,2, a}$ on behalf of the AMS Collaboration \\ ${ }^{1}$ INFN Sezione Perugia \\ ${ }^{2}$ Università degli Studi di Perugia
}

\begin{abstract}
The Alpha Magnetic Spectrometer, AMS-02, detector is operating on the International Space Station (ISS) since May the 19th, 2011. More than 80 billion events have been collected by the instrument in the first 5 years of data taking. This unprecedented amount of data is being used to perform accurate measurements of the different Cosmic Rays (CR) components. In this contribution a review of the published results will be presented.
\end{abstract}

\section{The AMS experiment and its scientific objectives}

The Alpha Magnetic Spectrometer, AMS-02, is a general purpose high energy particle physics detector. It was launched to space with the Space Shuttle STS-134 mission and installed onboard the ISS in 2011, on May 19th, to conduct a unique long duration mission (up to the lifetime of the ISS) of fundamental physics research in space. More than 80 billion events have been collected by the instrument in the first 5 years of data taking.

The experimental challenge of the experiment is the accurate measurement of the Charged Cosmic Rays (CCR) composition and energy spectra, up to the TeV scale, that could reveal the presence of primordial anti-matter or give the signature of 'exotic' sources. An accurate and high statistics measurement of all the various components is needed to have a better and coherent picture describing the observed CR phenomenology.

A good example to show the need of the full set of measurements is the indirect search of Dark Matter (DM). The annihilation of DM particles can produce matter-antimatter pairs (electron-positron, $e^{+}-e^{-}$, proton-antiproton, $p-\bar{p}$, etc...). These particles propagate through the Galaxy mixing up with the standard particles and can be revealed as excesses with respect to the expected fluxes. Rarer is the standard CR component (for example $e^{+}$and $\bar{p}$ ) and more statistical significant is any observed excess. The 'natural' candidate measurements where to search for excesses are the positron fraction, $e^{+} /\left(e^{-}+e^{+}\right)$, and the antiproton to proton ratio, $\bar{p} / p$.

To search for an excess with respect to an expected spectrum is obviously crucial to constrain as much as possible the models used to predict the purely primary or secondary spectra. The precise knowledge of the electron flux and its spectral features, for example, is mandatory to understand the features in the positron fraction, while the measurement of the proton flux and of the nuclear components (for

a e-mail: matteo.duranti@pg.infn.it 

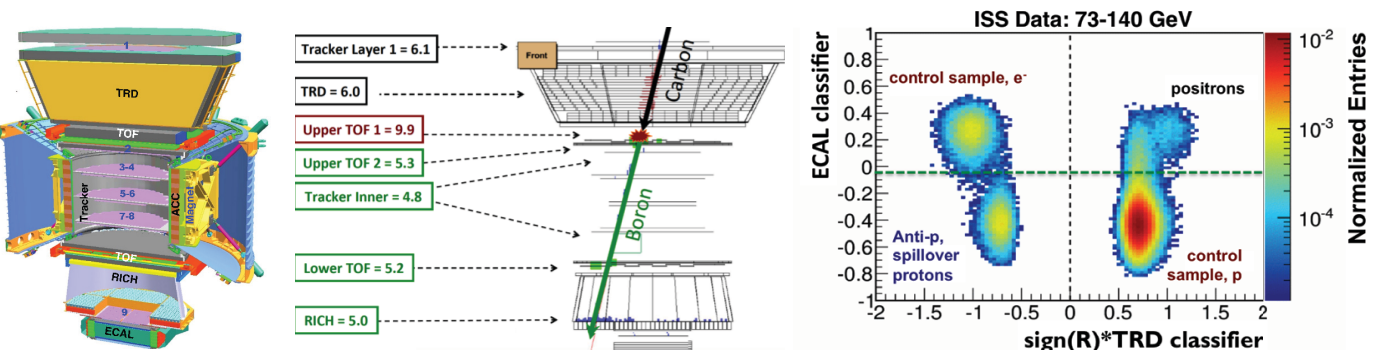

Figure 1. Left - Schematic view of the AMS-02 spectrometer. Center - A Carbon nucleus, fragmenting into a Boron one, as identified by the AMS detector on the ISS in the bending $(y-z)$ plane. Tracker planes measure the particle charge and momentum. The TRD measure the charge of the particle. The TOF measures the charge and ensures that the particle is downward-going. The RICH independently measures the charge and velocity. The discrepancy between the charge in the first layer of the Tracker and in the TRD with the one in the upper part of the TOF and in the remaining detectors, clearly identify the event as a Carbon nucleus fragmenting into a Boron one. Right - Distribution of the events, in the energy range 73-140 GeV, in the ECAL classifier - TRD classifier plane. Negative from positive events are distinguished by means of the sign of the Rigidity in the spectrometer, $\operatorname{sign}(R)$. The separation of the various components of $e^{-}, e^{+}, \mathrm{p}$ and $\bar{p}$ is clearly visible.

example the Boron/Carbon) is needed to predict the spectrum of the secondary positrons (from the collision of the CRs with the ISM) and their propagation through the Galaxy.

Unfortunately, at low energy, the spectra are also deformed by the effect of the solar wind. The solar modulation models are still quite rough or under development ([1-3]) and so a large campaign of measurement, for example of the fluxes as a function of time, can widely improve the models and the overall picture.

To reach its scientific goals, the AMS-02 instrument, whose schematic layout is reported in Fig.1 (left), has been conceived with a large redundancy in the measurement of particle properties with complementary techniques by different sub detectors. The apparatus is built around a magnetic spectrometer made of nine planes of precision silicon tracker and a permanent magnet. A Transition Radiation Detector (TRD), four planes of Time of Flight counters (TOF), an array of Anti-Coincidence Counters (ACC) surrounding the inner tracker, a Ring Imaging CHerenkov detector (RICH), and an Electromagnetic CALorimeter (ECAL) add completeness and redundancy to the whole detector.

Details about the performances of the different sub-detectors can be found in the first published result [4] and in the reference therein.

The identification of the various nuclear components is granted by the measurement of the charge of the impinging particles, all along the detector. Essentially all the sub-detectors have some charge measurement capability: the most accurate is the inner tracker, providing a $\sim 0.1$ c.u. charge resolution, with its 7 layers (each layer, alone, has a resolution of about 0.3 c.u.); the RICH and the TRD have a resolution of $\sim 0.3$ c.u., while each pair of planes of the TOF has a resolution of $\sim 0.16$ c.u.. This permits to follow the particle traversing the detector and to search for fragmentation that could spoil the charge reconstruction and constitute a source of background in the less abundant nuclear components. This is sketched in Fig.1 (center): a Carbon nucleus fragments into a Boron one interacting with the material of the upper pair of planes of the TOF (Upper TOF). The discrepancy between the charge measured by the first layer of the Tracker, L1, and the TRD, $\sim 6$, the one in the Upper TOF, $\sim 10$ (due to the large energy deposit of the interaction), and the one in all the remaining detectors, $\sim 5$, is a clear signature of fragmentation. The dynamic range of the various detectors permits, with a resolution slightly degrading with $Z$, the charge measurement up to the higher charges: Iron and 
above (the Zinc peak is clearly visible) for Tracker, TOF and RICH, and up to $Z \sim 6$ for the TRD. The discrimination power to the rare electromagnetic components, $e^{+}$and $e^{-}$, from the overwhelming background of protons, and from the even rarer antiprotons component is sketched in Fig.1 (right). The two key detectors to distinguish $e^{+} / e^{-}$from $\bar{p} / p$ are the ECAL (via a classifier exploiting the 3D imaging capability of the detector and built using a BDT approach [5]), and the TRD (via a loglikelihood to the energy deposit in its straw tubes). The discrimination between matter and anti-matter (i.e. $e^{+}$from $e^{-}$or $\bar{p}$ from $p$ ), is provided by the spectrometer. The redundancy is also a key capability that permits to build, for example using the TRD classifier and the sign of the rigidity, almost pure control samples of $e^{-}$and $p$, to study the performances of the ECAL.

The redundancy and the complementarity of the various sub-detectors of the apparatus, give to the experiment a full coverage of the CCR, both in terms of composition and spectral features.

\section{Experimental results}

The analysis of the enormous amount of data being collected by the experiment, permitted the publication of high statistics and very accurate measurement of the more important CCR components:

- $e^{+} /\left(e^{+}+e^{-}\right)$in the range $0.5-500 \mathrm{GeV},[4,6]$, based on 30 months and $\sim 10.9$ million positron and electron events;

- positron flux in the range $0.5-500 \mathrm{GeV}$ and electron flux in the range $0.5-700 \mathrm{GeV}$, [7], based on 30 months and $\sim 0.6$ million positrons and $\sim 9.2$ million electrons ;

- all-electron, $e^{+}+e^{-}$, flux, in the range $0.5-1000 \mathrm{GeV}$, [8], based on 30 months and $\sim 10.6$ million positron and electron events;

- $p$ flux in the range 1-1800 GV, [9], based on 30 months and 300 million protons;

- He flux in the range 1.9-3000 GV, [10], based on 30 months and 50 million helium events;

- antiproton flux and $\bar{p} / p$ ratio in the range $1-450 \mathrm{GV}$, [11], based on the first 4 years of the collected data, corresponding to $\sim 350000$ antiproton and $\sim 2.4$ billion proton events;

The high statistics, low systematics, measurement of the $e^{+}$and $e^{-}$components, see Fig.2, allowed the determination, with very high accuracy, of the spectral features of the $e^{+} /\left(e^{+}+e^{-}\right)$and of the fluxes of the single components:

- the accuracy of the measurement of the high energy rise in the positron fraction (already seen by PAMELA, [12], and FERMI, [13]), allowed the systematic study of the slope, that was found to be consistent with a zero crossing, i.e. with a maximum in the positron fraction, at $\sim 275 \mathrm{GeV}$.

- both the electron and positron fluxes have a spectral index increasing with energy after few GeV's. The hardening of both spectra gives the hint that the rise in the positron fraction is not due to a lack of high energy electrons, since their spectrum is hardening, but to a fresh source of positrons;

- the absence of structures in the all-electron flux. The flux is smooth and well described by a single power law, after $\sim 30 \mathrm{GeV}$ and doesn't show the peak claimed by ATIC, [14].

The measurement of the proton and helium fluxes, instead, not only permitted the confirmation of the spectral features already seen by PAMELA, [15], but, given the unprecedented accuracy, permitted a systematic study of the change in the spectral indexes, allowing the determination of the $\Delta \gamma$ 's and of the transition rigidity values, $R_{0}$, reported in Fig. 3 .

The measurement of the $\bar{p} / p$, finally, widely extended the experimental energy reach and the accuracy of the previous measurements, $[16,17]$ and resulted, at high energies, in a ratio almost independent from energy. 

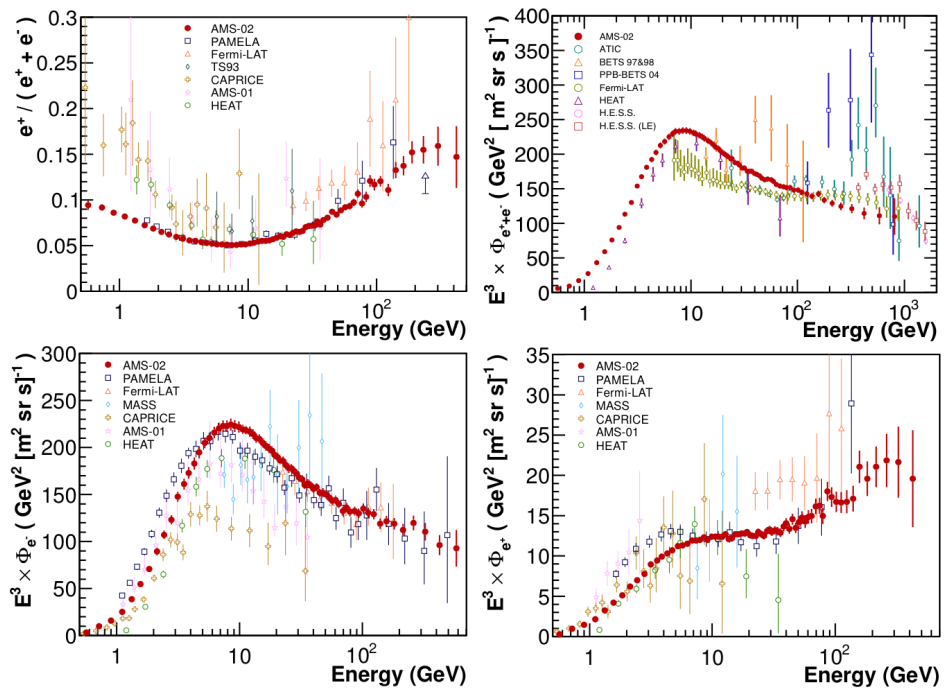

Figure 2. Positron fraction in the energy range $0.5-500 \mathrm{GeV}$ (top-left), all-electron flux in the range 0.5 $1000 \mathrm{GeV}$ (top-right), electron flux in the range $0.5-700 \mathrm{GeV}$ (bottom-left) and positron flux in the range 0.5 - $700 \mathrm{GeV}$ (bottom-right), as measured by the AMS02 experiment. The results from previous experiments are reported for comparison, [12, 14, 18-28].

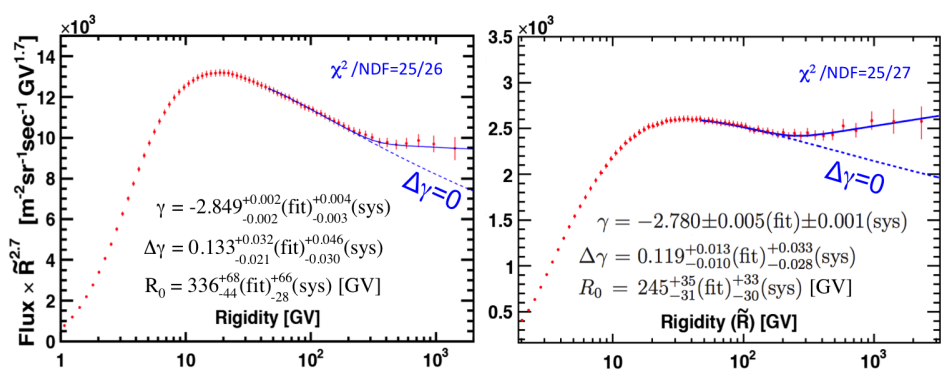

Figure 3. Left - Protons flux as measured by AMS- 02 up to $1.8 \mathrm{TeV}$. Right - Helium flux up to $3 \mathrm{TeV}$. The main parameters coming from the study of the spectral features are reported, together with the fit to the experimental points.

The big amount of collected data is being analyzed for the measurements of the other nuclear components ( $\mathrm{Li}, \mathrm{B}, \mathrm{C}, \mathrm{O}$ fluxes, $\mathrm{B} / \mathrm{C}, \mathrm{C} / \mathrm{O}$, etc..) and for the study of the other characteristics of the already published spectra (for example the variability of the electron, positron, proton, helium and antiproton fluxes and ratios as a function of time).

\section{Conclusions}

Data recorded in the first $\sim 5$ years of mission are being analyzed to cover the physics program of the AMS-02 experiment: all the measurements performed so far show interesting features, not expected 

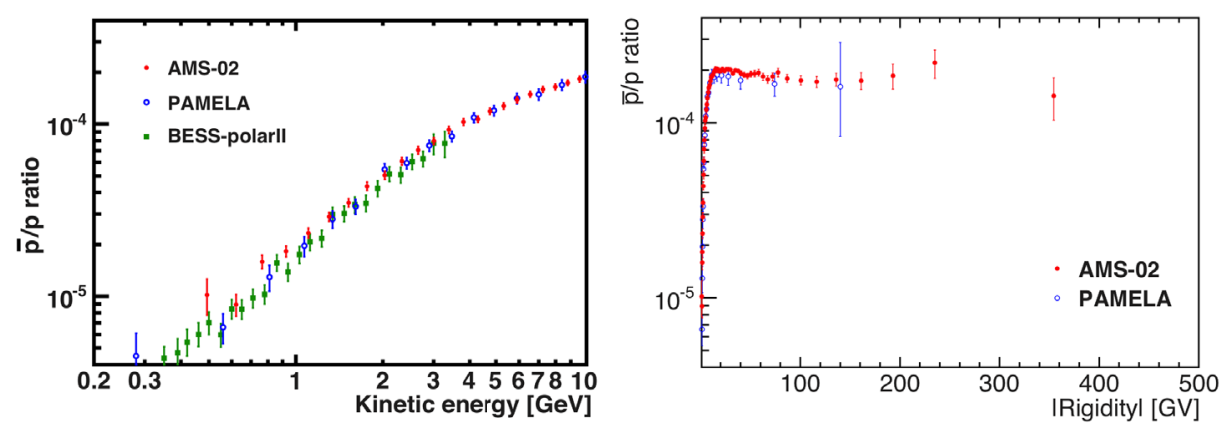

Figure 4. $\bar{p} / p$ ratio as a function of the Rigidity (on the right) and of the Kinetic Energy (for low energies, on the left). At high rigidities the ratio is almost independent from the energy. Results from previous experiments reported for comparison, [16, 17, 29].

by the standard model of CRs, extending previous observations and adding new findings whose experimental accuracy challenges the actual theoretical predictions. AMS-02 represents the first instrument with the capability to simultaneously study all the different nuclear CR species, including the less abundant ones and up to Iron, electrons and anti-matter with unprecedented accuracy and in an extended energy range. These unique features will allow AMS-02 to shed light on new phenomena and improve the current understanding of CR origin and propagation.

\section{Acknowledgments}

This work has been supported by acknowledged persons and institutions in the AMS-02 publications [4, 6-11], as well as by the Italian Space Agency under ASI-INFN Agreement No. 2013-002-R.0.

\section{References}

[1] L.J. Gleeson, W.I. Axford, Astrophysical Journal 154, 1011 (1968)

[2] M.S. Potgieter, Living Reviews in Solar Physics 10, 3 (2013)

[3] M. Gervasi, P. Rancoita, I. Usoskin, G. Kovaltsov, Nuclear Physics B - Proceedings Supplements 78, 26 (1999)

[4] M. Aguilar, G. Alberti, B. Alpat, A. Alvino, G. Ambrosi, K. Andeen, H. Anderhub, L. Arruda, P. Azzarello, A. Bachlechner et al. (AMS Collaboration), Phys. Rev. Lett. 110, 141102 (2013)

[5] B.P. Roe, H.J. Yang, J. Zhu, Y. Liu, I. Stancu, G. McGregor, Nuclear Instruments and Methods in Physics Research Section A: Accelerators, Spectrometers, Detectors and Associated Equipment 543, 577 (2005)

[6] L. Accardo, M. Aguilar, D. Aisa, B. Alpat, A. Alvino, G. Ambrosi, K. Andeen, L. Arruda, N. Attig, P. Azzarello et al. ((AMS Collaboration)), Phys. Rev. Lett. 113, 121101 (2014)

[7] M. Aguilar, D. Aisa, A. Alvino, G. Ambrosi, K. Andeen, L. Arruda, N. Attig, P. Azzarello, A. Bachlechner, F. Barao et al. ((AMS Collaboration)), Phys. Rev. Lett. 113, 121102 (2014)

[8] M. Aguilar, D. Aisa, B. Alpat, A. Alvino, G. Ambrosi, K. Andeen, L. Arruda, N. Attig, P. Azzarello, A. Bachlechner et al. ((AMS Collaboration)), Phys. Rev. Lett. 113, 221102 (2014)

[9] M. Aguilar, D. Aisa, B. Alpat, A. Alvino, G. Ambrosi, K. Andeen, L. Arruda, N. Attig, P. Azzarello, A. Bachlechner et al. (AMS Collaboration), Phys. Rev. Lett. 114, 171103 (2015) 
[10] M. Aguilar, D. Aisa, B. Alpat, A. Alvino, G. Ambrosi, K. Andeen, L. Arruda, N. Attig, P. Azzarello, A. Bachlechner et al. (AMS Collaboration), Phys. Rev. Lett. 115, 211101 (2015)

[11] M. Aguilar, L. Ali Cavasonza, B. Alpat, G. Ambrosi, L. Arruda, N. Attig, S. Aupetit, P. Azzarello, A. Bachlechner, F. Barao et al. (AMS Collaboration), Phys. Rev. Lett. 117, 091103 (2016)

[12] O. Adriani, G.C. Barbarino, G.A. Bazilevskaya, R. Bellotti, M. Boezio, E.A. Bogomolov, L. Bonechi, M. Bongi, V. Bonvicini, S. Bottai et al., Nature 458, 607 (2009)

[13] M. Ackermann, M. Ajello, A. Allafort, W.B. Atwood, L. Baldini, G. Barbiellini, D. Bastieri, K. Bechtol, R. Bellazzini, B. Berenji et al. (Fermi LAT Collaboration), Phys. Rev. Lett. 108, 011103 (2012)

[14] J. Chang, J.H. Adams, H.S. Ahn, G.L. Bashindzhagyan, M. Christl, O. Ganel, T.G. Guzik, J. Isbert, K.C. Kim, E.N. Kuznetsov et al., Nature 456, 362 (2008)

[15] O. Adriani, G.C. Barbarino, G.A. Bazilevskaya, R. Bellotti, M. Boezio, E.A. Bogomolov, L. Bonechi, M. Bongi, V. Bonvicini, S. Borisov et al., Science 332, 69 (2011)

[16] O. Adriani, G.A. Bazilevskaya, G.C. Barbarino, R. Bellotti, M. Boezio, E.A. Bogomolov, V. Bonvicini, M. Bongi, L. Bonechi, S.V. Borisov et al., Soviet Journal of Experimental and Theoretical Physics Letters 96, 621 (2013)

[17] O. Adriani, G.C. Barbarino, G.A. Bazilevskaya, R. Bellotti, M. Boezio, E.A. Bogomolov, L. Bonechi, M. Bongi, V. Bonvicini, S. Bottai et al., Physical Review Letters 102, 051101 (2009), Q810. 4994

[18] O. Adriani, G.C. Barbarino, G.A. Bazilevskaya, R. Bellotti, M. Boezio, E.A. Bogomolov, M. Bongi, V. Bonvicini, S. Borisov, S. Bottai et al., Phys. Rev. Lett. 106, 201101 (2011)

[19] M. Ackermann, M. Ajello, W.B. Atwood, L. Baldini, J. Ballet, G. Barbiellini, D. Bastieri, B.M. Baughman, K. Bechtol, F. Bellardi et al. (Fermi LAT Collaboration), Phys. Rev. D 82, 092004 (2010)

[20] J.J. Beatty, A. Bhattacharyya, C. Bower, S. Coutu, M.A. DuVernois, S. McKee, S.A. Minnick, D. Müller, J. Musser, S. Nutter et al., Phys. Rev. Lett. 93, 241102 (2004)

[21] F. Aharonian, A.G. Akhperjanian, U. Barres de Almeida, A.R. Bazer-Bachi, Y. Becherini, B. Behera, W. Benbow, K. Bernlöhr, C. Boisson, A. Bochow et al. (H.E.S.S. Collaboration), Phys. Rev. Lett. 101, 261104 (2008)

[22] Aharonian, F., Akhperjanian, A. G., Anton, G., Barres de Almeida, U., Bazer-Bachi, A. R., Becherini, Y., Behera, B., Bernlöhr, K., Bochow, A., Boisson, C. et al., A\&A 508, 561 (2009)

[23] R.L. Golden, S.J. Stochaj, S.A. Stephens, F. Aversa, G. Barbiellini, M. Boezio, U. Bravar, A. Colavita, F. Fratnik, P. Schiavon et al., Astrophysical Journal Letters 457, L103 (1996)

[24] M. Boezio, P. Carlson, T. Francke, N. Weber, M. Suffert, M. Hof, W. Menn, M. Simon, S.A. Stephens, R. Bellotti et al., The Astrophysical Journal 532, 653 (2000)

[25] M. Aguilar, J. Alcaraz, J. Allaby, B. Alpat, G. Ambrosi, H. Anderhub, L. Ao, A. Arefiev, P. Azzarello, L. Baldini et al., Physics Letters B 646, 145 (2007)

[26] S. Torii, T. Tamura, N. Tateyama, K. Yoshida, J. Nishimura, T. Yamagami, H. Murakami, T. Kobayashi, Y. Komori, K. Kasahara et al., The Astrophysical Journal 559, 973 (2001)

[27] K. Yoshida, S. Torii, T. Yamagami, T. Tamura, H. Kitamura, J. Chang, I. Iijima, A. Kadokura, K. Kasahara, Y. Katayose et al., Advances in Space Research 42, 1670 (2008)

[28] C. Grimani, S.A. Stephens, F.S. Cafagna, G. Basini, R. Bellotti, M.T. Brunetti, M. Circella, A. Codino, C. De Marzo, M.P. De Pascale et al., Astronomy and Astrophysics 392, 287 (2002)

[29] K. Abe, H. Fuke, S. Haino, T. Hams, M. Hasegawa, A. Horikoshi, K.C. Kim, A. Kusumoto, M.H. Lee, Y. Makida et al., Physical Review Letters 108, 051102 (2012), 1107.6000 